\title{
Multiple shear bands in granular materials
}

\author{
Sára Lévay ${ }^{1, \star}$ and János Török ${ }^{1}$ \\ ${ }^{1}$ Department of Theoretical Physics, Budapest University of Technology and Economics, $\mathrm{H}-1111$ Budapest, Hungary
}

\begin{abstract}
The transition regime from quasi-static to dynamic shear regime is studied by discrete element method $(D E M)$ and by means of a mesoscopic model. We show that at moderate shear rate multiple shear bands appear which eventually appear as a continuous shear profile for large shear rates. The model and the DEM simulations both show a minimum in the shear stress and a maximum for the number of simultaneous shear bands, however the position of the two do not coincide. The scaling analysis of the kinetic energy indicates that the peak in the latter is the relevant parameter. We also show how the long range order present in the quasi-static regime is gradually destroyed by the presence of multiple shear bands.
\end{abstract}

\section{Motivation}

Granular shear is a very complex process [1-3] with different characteristics according to the shear velocity [4] or the properties of the sheared material itself, e.g. wet or dry [5]. Here we only focus on cohesionless materials where still there is a qualitative difference in the behavior of the sample depending on the imposed strain rate. The slowly sheared granular material show intermittent shear characterized by shear bands, while fast shear results in a continuous shear velocity profile [6]. In this paper we will focus on the crossover of these two processes.

Slow or quasi-static shear is characterized by a slow increase of the stress which eventually released in a plastic event which is in general a shear band compatible with the boundary conditions. The plastic process restructures the material and if large strains are used the above process is repeated indefinitely $[7,8]$ : The stress increases again and gets released in a new plastic event. In general the position of the plastic event is determined by inhomogeneities of the material, however the structure of the material is modified by the plastic event. This creates a self-organized process which may result in long range correlations [7, 9].

In this paper we study the crossover from slow to fast shear of granular materials by performing discrete element simulation and presenting a mesoscopic model describing this. We show how long range correlations built up by the slow shear are destroyed by fast shear.

\section{Discrete Element Method}

\subsection{Simulation}

Discrete Element Method (DEM) simulations were performed to study the shear of granular materials. We used the molecular dynamics code LAMMPS [10] to determine

\footnotetext{
^e-mail: levay.sara93@gmail.com
}
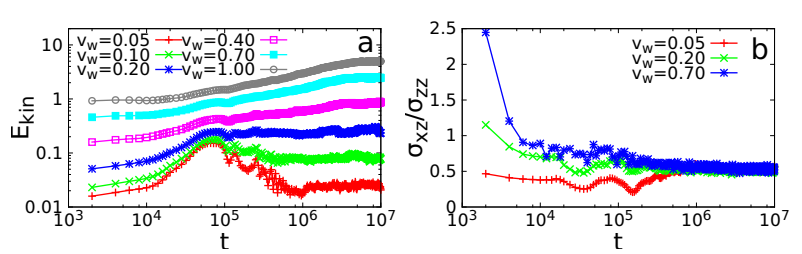

Figure 1. The time evolution of (a) the total kinetic energy and (b) the relative shear stress.

the crossover from slow to fast shear. Two-dimensional plane shear geometry was created where the granular material is placed between two rough walls in the $x z$ plane. The walls are held together with confining stress $\sigma_{z z}$. The walls in our simulations were build up from randomly placed spherical particles with diameters 1.4 times larger than the average particle size to create as large wall friction as one can get [11]. We created polydisperse samples with the variation of particle diameters by $\pm 20 \%$. The mean particle diameter was chosen to be the distance unit. The coefficient of friction between particles was $\mu=0.3$. The initial configurations were created by compressing samples of random sequential deposition by the confining walls. After the system is relaxed, walls start to move in direction $x$ with constant velocities $\left(v_{w}\right)$ equal in magnitude but opposite in direction. We apply periodic boundary conditions in the $x$ direction. For each wall velocity 15 independent configurations were sheared for $10^{5} \mathrm{DEM}$ time units and the results show ensemble and time averages of these simulations. The natural units of the DEM simulation are $d=1, \rho=1$ and $\sigma_{z z}=1$, particle stiffness was $k=10^{4}$. 


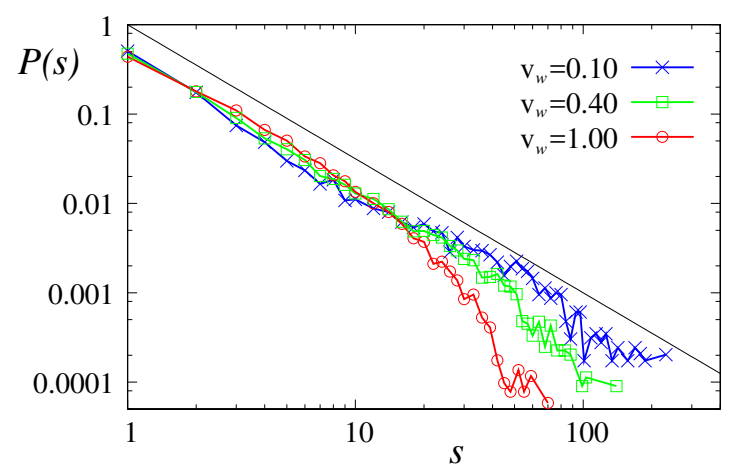

Figure 2. Avalanche size distribution for different values of wall velocity. The solid line is only to drive the eye.

\subsection{Results}

Due to self-organized criticality quasi-static granular shear can build up long range correlations that span the entire system. There are many signs in the system which account for long range order: The non-monotonic behavior of the system in the transient [12] or the presence of critical exponents [13]. Therefore we start our investigation by testing this assumption. In the DEM simulations we measure the shear stress and the total kinetic energy as function of the shear strain. Fig. 1 shows the time evolution of the latter two quantities for different wall velocities. It is obvious that the transient behavior of both $\sigma_{x z}$ and $E_{\mathrm{kin}}$ is non-monotonic for wall speeds up to $v_{\text {wall }} \simeq 0.3$ but above this threshold, simple relaxation can be seen.

Many critical exponents can be defined for sheared granular material [14], here we restrict ourselves to the study of avalanche size distribution $P(s)$ presented in Fig. 2 and Fig. 5. An avalanche is characterized by a series of correlated plastic events and can measured by correlations of the shear stress fluctuations. We define the length of an avalanche by the time interval between successive passes of the shear stress though given level [15]. The resulting avalanche size distribution is a power law for slow shear. As the wall velocity increases an exponential cutoff appears at earlier and earlier stages. Similar behavior was already found in [13].

The question we would like to answer is what causes the disappearance of the long range correlations for quicker shear. To answer this question we measured instantaneous velocity profiles. Four samples are shown in Fig. 3 for inertial number is $I=\dot{\gamma} d / \sqrt{P / \rho}=2.45 \cdot 10^{-2}$ and $I=2.45 \cdot 10^{-3}$ ( $\dot{\gamma}$ is the shear rate, $d=1$ is the average particle diameter, $P=1$ is the pressure and $\rho=1$ is the density). In the case of large inertial number the shear profile is linear [Fig. 3 (d)], but if the inertial number is slightly above the quasi-static limit of $I=2.45 \cdot 10^{-3}>10^{-3}$ we see a varying number of shear bands in the sample [Figures 3 (a)-(c)]. We believe that the presence of multiple shear bands is responsible for the appearance of a finite correlation length and the collapse of the scaling quasistatic regime.
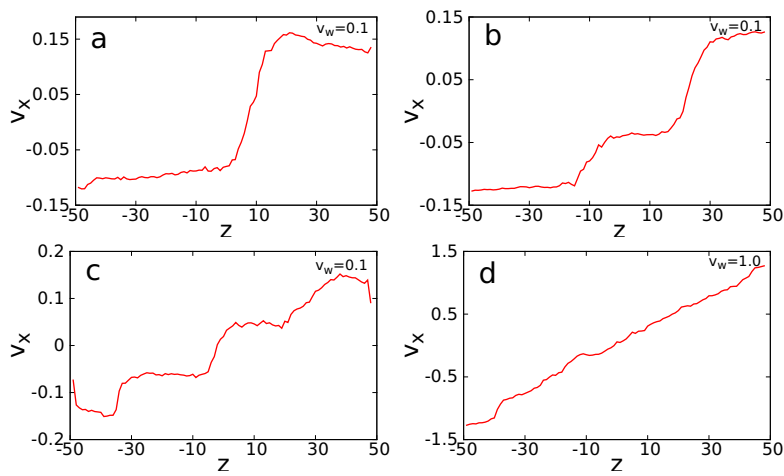

Figure 3. Sample velocity profiles at different time positions for the discrete element method with (a)-(c): $v_{w}=0.1$, and (d) $v_{w}=1.0$.

\section{Mesoscopic model}

\subsection{Description}

In order to have a better understanding of the system we introduce a simple mesoscopic model motivated by the above observations which can describe simple shear for different shear rates. Dry granular materials are best described by Coulomb-friction. The material is stable for shear stress up to $\sigma_{x z}<\mu \sigma_{z z}$, where $\sigma_{x z}$ is the shear stress, $\sigma_{z z}$ is the confining stress and $\mu$ is the effective mesoscopic friction coefficient. In this publication we suppose that the shear rate is still relatively low and the change of volume fraction and friction coefficient with shear rate can be neglected.

We discretize the system on a mesoscopic scale which is taken to be the average particle diameter $\langle d\rangle$. Due to symmetry reasons the simple shear setup can be described by a one-dimensional system in the $z$ direction. Thus the whole system is represented by a one-dimensional lattice where each site is characterized by a single static parameter the effective friction coefficient.

Shear stress, which increases due to wall displacement, will be released by localized plastic events which are the shear bands. Shear bands will appear at positions where the shear stress surpasses the local Coulomb limit. The observed macroscopic shear stress is determined from these local plastic events. The shear band, which is a dynamic event, has a timescale $\tau_{0}$, and an effect on the local material properties which we consider here completely random. Thus the material will be modeled by local randomization of the effective friction coefficient at the shear band and in the neighboring sites. This local randomization is the essence of our mesoscopic model. In this publication for simplicity we always use uniform random numbers between 0 and 1 .

Wall velocity in this mesoscopic picture will be represented by $\Delta$ which is the shear stress increase in a unit time which is the characteristic timescale of a shear band $\tau_{0}$. We further assume that the stress redistribution is fast compared to $\tau_{0}$ which is in line with our previous assumptions that the shear is not yet in the fully dynamic regime. 

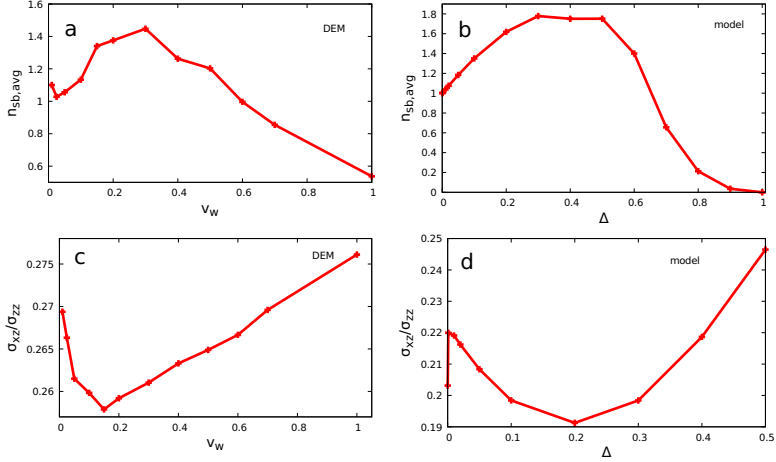

Figure 4. Average number of shear bands in (a) DEM simulation as function of the wall velocity and in (b) mesoscopic model as function of $\Delta$. Average shear stress in (c) DEM simulation as function of the wall velocity and in (d) mesoscopic model as function of $\Delta$.

In summary our mesoscopic model is defined as follows: The system is characterized by a one-dimensional mesoscopic system due to symmetries of the setup. The material is described by a single scalar: the local effective friction coefficient. The shear stress in the material is homogeneous and is increased by $\Delta$ during the timescale $\tau_{0}$. All positions for which the shear stress exceeds the local Coulomb threshold, the material fails and a local shear band develops. The local shear band reorganizes the material under and in the immediate vicinity of the shear band modeled by random change of the local effective friction coefficient. With the plastic events the stress is released from the system but again it will be increased in steps of $\Delta$.

\subsection{Results}

In Fig. 4 (a)-(b) we measured $n_{s b}$ the average number of simultaneous shear bands both in the DEM simulation and the mesoscopic model. In the simulation a shear band was defined as follows: The strain rate at a given point was defined by the local velocity difference which was measured at 5 particle diameter distance to reduce error from fluctuations. The the average $\gamma_{a}$ and the maximal $\gamma_{m}$ local strain rate was determined. The value of $\gamma_{m}$ was limited to $3 \gamma_{a}$, to disregard excess fluctuations. A threshold was set to be $\gamma_{t}=\left(\gamma_{m}+\gamma_{a}\right) / 2$, halfway between the maximum and the average. The number of shear bands $S$ is defined by the number of intervals where the local shear rate exceeds the threshold $\gamma_{t}$. Only configurations with plastic event were considered.

In the mesoscopic model it is not enough to calculate the number of failed sites to have the number of shear bands if we want to compare it to DEM simulation. Failed sites next to each other appear as one single shear band in DEM data. Therefore in the mesoscopic model we imitated this and we counted clustered shear bands as one.

Clustering adjacent shear bands has the important characteristics that one should observe a peak at the transition, since on either limits of global shear rate the value of $n_{s b}$ is small: For low shear rates there is only one shear band, for very large shear rate there is shear band everywhere so it is just a continuous homogeneous shear for which our method gives $n_{s b}=0$. In between there are cases with higher values of $n_{s b}>1$.

The resulting curves in Fig. 4 (a)-(b) show the predicted feature: the curve start from $n_{s b}=1$ there is a maximum at $v_{w}=0.3$ and at $\Delta=0.4$ and for large shear $n_{s b}$ drops well below one. This result affirms the appearance of multiple shear bands in the transitional regime.

Let us note that in a completely different field of opinion dynamics in a collaborative environment similar transition was found. The crossover is between a step-wise function and an overall increasing one with maximum density of steps at the transition [16].

The average shear stress $\sigma_{x z} / \sigma_{z z}$ in Fig. 4 (c)-(d) shows also interesting features. It has a minimum at lower values of $v_{w}$ and $\Delta$ than the position of the maximum of the number of shear bands. The presence of a minimum in the macroscopic shear stress is counterintuitive as in general the shear stress increases with the shear rate [1]. It is thus surprising that we see an opposing trend for low wall velocities. The model can give an understanding of this feature.

In our model a shear band restructures three sites, the one under the shear band and the two neighboring ones. Since this is done uncorrelatedly of the previous state these local friction coefficients will be random. So in the next step the weakest one has a value which is the minimum out of 3 independent random numbers. In our case it is 1/4. In Fig. 4 (d) the obtained value for $\Delta \simeq 0$ is somewhat smaller which is due to the fact that the minimal site may jump to previous positions of the current avalanche.

The number of refreshed sites increases with the number of shear bands so a smaller minimum can be chosen. There is however a counter effect that not only the minimum but all sites in a $\Delta$ interval fail thus increasing the shear stress which effect eventually wins and increases the stress rate. At very high shear rates no minimization takes place as almost every site is a shear band.

In DEM simulations the same effect may happen, namely that if sheared moderately fast, then the multiple shear band weaken the system which can be sheared easier. On the other hand faster shear requires too many shear bands which does not allow for any optimization. Of course for even faster shear dynamic effects become more important and the shear stress will increase further.

Furthermore the correspondence between the model and the simulation indicates that the duration of a shear band is $\tau_{0} \simeq 1$ which is supported by our preliminary results in DEM simulations.

The above observation helps us understand the difference between the position of the minimum in the macroscopic stress and the number of shear bands as the previous one incorporates two effects in a nontrivial way: the number of shear bands and the stress increase in unit time.

The kinetic energy in the steady state is only available in the DEM simulation. Figure 6 shows $E_{\text {kin }} / v_{w}^{2}$ as function $v_{w}$. For large wall velocities the velocity profile of the 


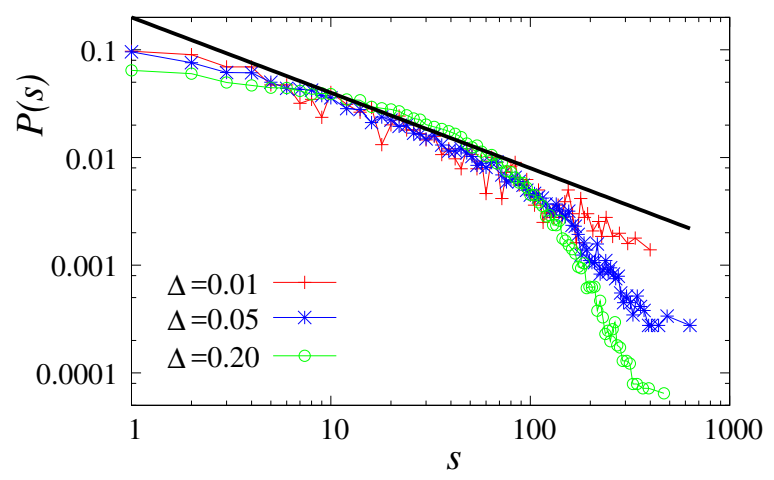

Figure 5. Avalanche size distribution for different values of $\Delta$ for the mesoscopic model. The solid line is only to drive the eye.

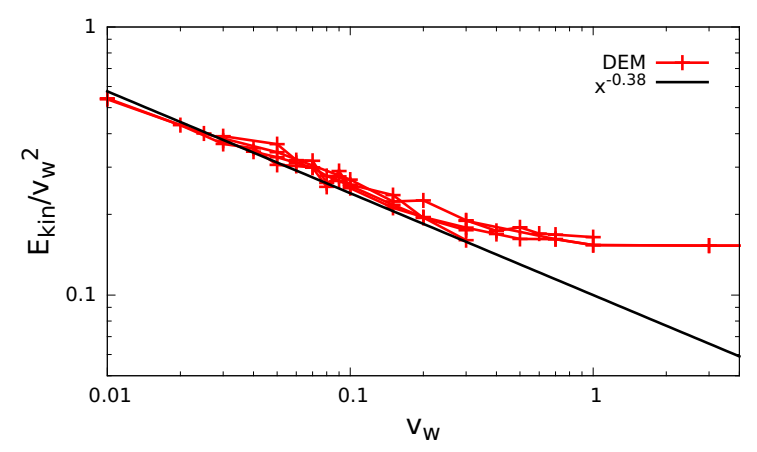

Figure 6. The kinetic energy rescaled with the square of the wall velocity as function of the wall velocity.

material is linear so the total kinetic energy stored in the system should be proportional to the square of the wall velocity. This is shown by the constant value for large $v_{w}$. On the other hand, for small wall velocities we have seen a power law increase which indicates super linear velocity profiles [17] which were found in quasi-static shear. There is a nice crossover between the two regimes at around $v_{w} \simeq 0.3$ which coincides with the maximum of the number of shear bands rather than with the minimum of the shear stress.

This latter finding indicates that the transition from quasi-static to dynamic regime is driven by multiple shear bands. The minimum of the shear stress is most probably the result of the crossover of two effects: one which decreases the shear stress (parallel shear bands in the avalanche), and an other one which is the mobilization of more stable regimes.

\section{Summary}

In this paper we have introduced a mesoscopic model for relatively slow granular shear. We studied the transition from quasi-static to dynamic shear. The model shows surprisingly similar features to the DEM simulations, namely there is a minimum in the shear stress and a maximum at the number of simultaneous shear bands at larger wall velocity. The long range order in quasi-static regime man- ifested in the power law distribution of avalanches is gradually destroyed by the presence of multiple shear bands in both cases. This indicates that real quasi-static system in infinite samples can be observed only by infinitely slow shear. The crossover in the kinetic energy suggests that the number of shear bands is the most important indicator of the transition to the fully dynamic regime.

It is still an open question how the presence of multiple shear bands is able to lower the average shear stress of the material, and what is the effect of the system size for the above quantities.

\section{Acknowledgements}

Support from the ÚNKP-16-2-I. New National Excellence Program of the Ministry of Human Capacities and from the Hungarian National Research, Development and Innovation Office NKFIH under grant OTKA K116036 is acknowledged.

\section{References}

[1] G. MiDi, The European Physical Journal E 14, 341 (2004)

[2] P.C. Johnson, R. Jackson, Journal of Fluid Mechanics 176, 67 (1987)

[3] C.S. Campbell, Annual Review of Fluid Mechanics 22, 57 (1990)

[4] T. Börzsönyi, R.E. Ecke, J.N. McElwaine, Physical Review Letters 103, 178302 (2009)

[5] V. Richefeu, M.S. El Youssoufi, F. Radjai, Physical Review E 73, 051304 (2006)

[6] Z. Shojaaee, J.N. Roux, F. Chevoir, D.E. Wolf, Physical Review E 86, 011301 (2012)

[7] L. Bocquet, A. Colin, A. Ajdari, Physical Review Letters 103, 036001 (2009)

[8] M. Manning, J. Langer, J. Carlson, Physical Review E 76, 056106 (2007)

[9] M. Talamali, V. Petäjä, D. Vandembroucq, S. Roux, Comptes Rendus Mecanique 340, 275 (2012)

[10] S. Plimpton, P. Crozier, A. Thompson, Sandia National Laboratories 18 (2007)

[11] Z. Shojaaee, L. Brendel, J. Török, D.E. Wolf, Physical Review E 86, 011302 (2012)

[12] B. Szabó, J. Török, E. Somfai, S. Wegner, R. Stannarius, A. Böse, G. Rose, F. Angenstein, T. Börzsönyi, Physical Review E 90, 032205 (2014)

[13] K.A. Dahmen, Y. Ben-Zion, J.T. Uhl, Nature Physics 7, 554 (2011)

[14] K.M. Salerno, C.E. Maloney, M.O. Robbins, Physical Review Letters 109, 105703 (2012)

[15] P. Bak, K. Sneppen, Physical Review Letters 71, 4083 (1993)

[16] J. Török, G. Iñiguez, T. Yasseri, M. San Miguel, K. Kaski, J. Kertész, Physical Review Letters 110, 088701 (2013)

[17] F. Da Cruz, F. Chevoir, J. Roux, I. Iordanoff, Tribology Series 43, 53 (2003) 